\title{
$\mathrm{TiO}_{2}$ 超微粒的毛细作用排列 ${ }^{*}$
}

\author{
顾 宁沈耀春 韦齐和陆祖宏韦钰 \\ (东南大学吴健雄实验室, 南京 210096)
}

\section{关钺词 $\mathrm{TiO}_{2}$ 超微粒 (UFP) 毛细作用 原子力显微镜 (AFM)}

超微粒子 (UFP) 的介观特性突出表现为量子限域的性质, 其最基本的原因在于 “尺寸效 应” 和 “表面效应”. 当粒子尺寸减小到可与电子的 de Broglie 波长 (约 $12 \mathrm{~nm}$ ) 相比拟或比其 更小时, UFP 成为所谓的量子点. 从量子理论的基本原理出发, 依据理想的量子点模型进行 加工, 可构造出新一代的量子电子器件 ${ }^{[1]}$. 出于与理论模型设计进行相互验证, 以及实际制作 新型器件的需要, 研究者们希望能将这些量子点进一步形成为一维或二维的量子点规则排 列, 成为某种超结构 ${ }^{[2]}$.

二氧化钩 $\left(\mathrm{TiO}_{2}\right)$ 是一种重要的宽禁带宽度的半导体光电转换材料, 广泛地用作为光阳极 催化分解水、太阳能电池等光化学以及光电子学器件的功能材料 ${ }^{[3]}$. 此外, 由于 $\mathrm{TiO}_{2}$ 薄膜具有 相当大的比表面积, 尤其是当 $\mathrm{TiO}_{2}$ 粒子粒径减至纳米量级时, 极易吸附气体, 并由此明显地改 变材料的导电性能, 故又被用于气体传感器的研制 ${ }^{[4]}$. 制备 $\mathrm{TiO}_{2}$ 薄膜的方法很多, 例如, 等离 子体增强化学气相沉积 (PECVD) 技术 ${ }^{[}$、旋转涂覆以及 Langmuir-Blodgett(LB) 方法 ${ }^{10}$ 等. 其 中, 利用硬脂酸 $(\mathrm{StA})$ 单分子层铺覆于含四氯化钝 $\left(\mathrm{TiCl}_{4}\right)$ 水溶液的 $\mathrm{LB}$ 槽中进行化学反应吸 附, 然后再转移成膜的 LB 法有可能形成 $\mathrm{TiO}_{2}$ UFP 的有序结构排列 ${ }^{17}$, 不过此时 $\mathrm{TiO}_{2}$ 已包裹 了一种高极性的偶电层, 这种表面修饰必然影响 $\mathrm{TiO}_{2}$ UFP 的电子能级结构. 为了使裸露 $\mathrm{TiO}_{2}$ UFP 能在一定基底上有序排列, 我们尝试利用表面毛细作用来驱使 UFP 在粗粘度为原 子级的表面上进行排列, 采用原子力显微镜 (AFM) 扫描观察排列情况, 获得了一些很有意义 的结果.

\section{1 实验}

\section{$1.1 \mathrm{TiO}_{2} \mathrm{UFP}$ 胶体溶液的制备}

$\mathrm{TiO}_{2}$ UFP 的制备方法已有一些报道 ${ }^{\left[{ }^{8}\right.}$. 在本文中, 我们通过水解针酸四丁酯 $\left(\left(\mathrm{C}_{4} \mathrm{H}_{8} \mathrm{O}\right)_{4} \mathrm{Ti}\right)$ 来制备 $\mathrm{TiO}_{2}$ UFP 的胶体溶液, 其具体过程已在前文中进行过介绍 ${ }^{6}$. 实验中, 所用铜酸 四丁酯、异丙醇 $\left(\left(\mathrm{CH}_{3}\right)_{2} \mathrm{CHOH}\right)$ 、硝酸等皆为分析纯. 去离子水经过两次重蒸馏, 所获溶液为 略呈乳白色的透明胶体溶液. 该 $\mathrm{TiO}_{2}$ UFP 胶体溶液十分稳定, 在室温下至少可保存放置 6 个月. 根据不同的制膜需要, 可采用真空旋转蒸发浓缩或加去离子水稀释的方法配制所需浓 度的 $\mathrm{TiO}_{2}$ UFP 溶胶液.

\footnotetext{
1995-02-08 收稿, 1995-05-09 收修改稿
}

*国家自然科学基金资助项目 


\section{$1.2 \mathrm{HOPG}$ 上 $\mathrm{TiO}_{2}$ UFP 膜的制备}

选择制膜的基底为高取向热解石墨 (HOPG). 每次制样前都将 HOPG 新解理一层, 随即 将薄膜制作于 $\mathrm{HOPG}$ 的新鲜表面上. 采用 $2 \mu \mathrm{L}$ 的微量进样器装载 $\mathrm{TiO}_{2}$ 浓度为 $0.5 \mathrm{~mol} / \mathrm{L}$ 的 UFP 溶胶液, 在 HOPG 表面选定位置处以充分接近方式落一滴, 可观察到溶胶液在 HOPG 表面迅即铺张开来. 待溶剂蒸发后即可获得一 定面积的 $\mathrm{TiO}_{2}$ UFP 薄膜. 此过程示意于图 1. 为了进行对比, 以同样的溶胶液采用旋涂法得到 $\mathrm{HOPG}$ 上的 $\mathrm{TiO}_{2}$ UFP 涂覆薄膜. 旋涂条件为 $800 \mathrm{r} / \mathrm{min}, 1 \mathrm{~min}$. 为简便分析, 所有样品均未 经过后烘, 全为室温条件下获得.

\section{3 采用 AFM 对样品表面结构进行观察}

微量进样器

对采用不同方法获得的 $\mathrm{TiO}_{2}$ UFP 膜进行

了 AFM 扫描观察. AFM 为美国 Digital Instrumets 公司产品, Nanoscope III 型, HOPG 亦由该公司购得. 观测中分别采用最大扫描范 围 $0.7 \mu \mathrm{m}$ 和 $12.5 \mu \mathrm{m}$ 的标准扫描头 $\mathrm{A}$ 和 $\mathrm{D}$. $\mathrm{Si}_{3} \mathrm{~N}_{4}$ 微悬臂取 $200 \mu \mathrm{m}$ 长型, 力弹性常数为 $0.12 \mathrm{~N} / \mathrm{m}$. 所有采集的图像均在恒斥力模式下取 得. Cantilever 与样品表面的作用力控制在 $10 \mathrm{nN}$ 左右. 所有的观测都在室温的大气环境 下进行.

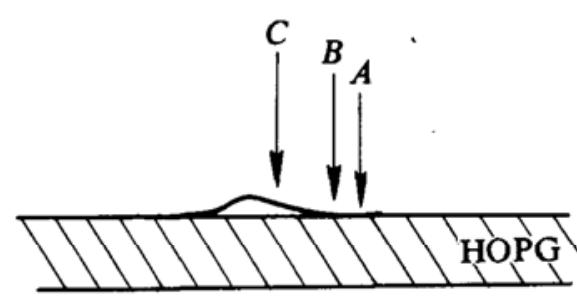

\section{2 结果与讨论}

\section{$2.1 \mathrm{TiO}_{2}$ UFP 溶胶液的铺展}

如图 1 所示, 将一滴(约 $0.2 \mu \mathrm{L}$ ) 的 $\mathrm{TiO}_{2}$ UFP 溶胶液滴于新解理的 HOGP 表面上, 肉眼 即可发现胶液在 HOPG 一定面积上迅速铺展开来. 若溶液量足够, HOPG 可为这种溶液充 分润湿, 可见该溶液与 HOPG 的接触角必为锐角. 这是因为, 尽管 HOPG 表面原本呈疏水 性, 但由于该水溶液中含有一定量的异丙醇, 使得混合液的表面张力大为减少, 进而使得 HOPG 可被其所润湿.

随着溶胶液的不断铺展, 同时溶液中溶剂不断蒸发, 使得 HOPG 上的液膜逐渐变得很 薄. 并且, 液膜越薄, 溶剂蒸发时间越短. 当液膜厚度小于胶溶液中粒子尺寸时, 粒子之间的 液面呈弯月面, 粒子间的作用主要为毛细作用. 相比较而言, 不考虑粒子之间的静电作用以 及基底上相对独立的 C 原子的力场等因素. 不过, 粒子仍然可能因具备热能而作 Brown 运 动, 发生碰撞等相互作用. Nagayama 等人对此情形曾进行过理论分析 ${ }^{[9]}$, 计算当液膜厚度小 于粒子尺度时, 对 $10 \mathrm{~nm}$ 粒径的粒子而言, 粒子间的毛细作用为其热运动作用能的 100 倍. 进 一步将其曲线外推得到, 当粒子径为 $2 \mathrm{~nm}$ 时, 毛细作用为热运动作用能的 10 倍左右. 当溶剂 以不断提高的速率继续蒸发过程中, 主导的毛细作用可迫使粒子按一定规则排列. 待溶剂蒸 发完之后, 即可得到排列于基底上的 UFP 膜. 


\section{2 毛细作用排列 $\mathrm{TiO}_{2}$ UFP 膜的 AFM 观察}

在制样前, 首先对新鲜的 HOPG 表面进行了 AFM 观察. 扫描采用 A 标准头, 扫描范围 为 $6.4 \mathrm{~nm} \times 6.4 \mathrm{~nm}$, 只经过滤除高频噪声的图象处理, 结果如图 2 所示. 图中 C 原子的直径约为 $0.3 \mathrm{~nm}$, 表面结构特征与 Tersoff 等人报道的结果相吻合 ${ }^{[10]}$. 由此可证实所采用的基底 HOPG 具有粗䊁度为原子级的表面.

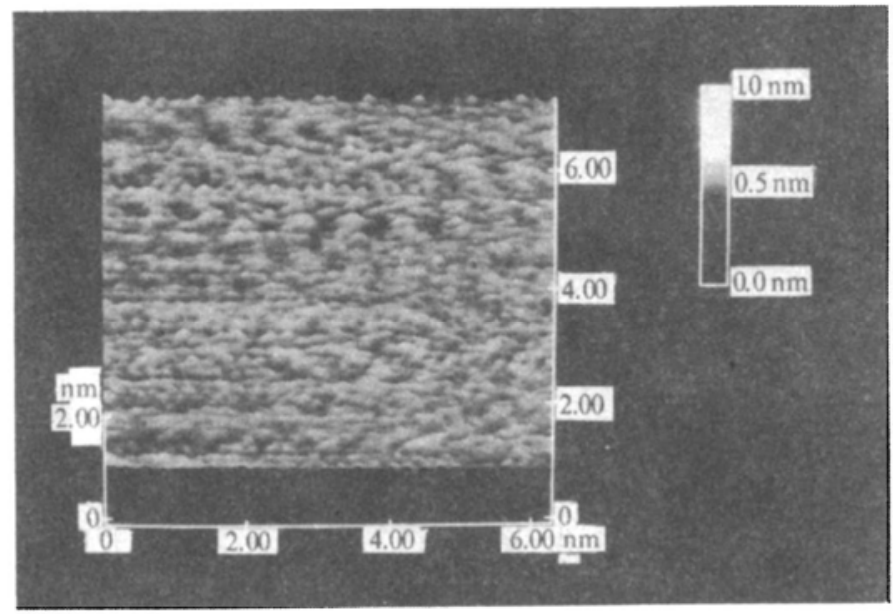

图 2 高取向热解石墨 (HOPG) 的新解理表面的 AFM 图象

样品的制备如图 1 所示. 在样品上距落滴处不同距离的 $A, B, C 3$ 个位置上(各自相距约 $2 \sim 3 \mathrm{~mm}$ ) 分别进行 AFM 扫描观察. 在此过程中发现, 样品必须待表面上溶剂充分蒸发后 才可用作分析, 因为表面上非常少量的蒸发都将扰乱 AFM 中的反射激光束斑, 使数据采集无 法进行. 实际工作中, $\mathrm{AFM}$ 分析一般待落滴后 $2 \mathrm{~h}$ 左右进行. $A, B, C$ 三点扫描所获得的 AFM 图象列为图 3(a) (c). 由图 3(a) 可见 $A$ 处的 $\mathrm{TiO}_{2}$ UFP 的排列情况, 扫描范围为 $13 \mathrm{~nm} \times 13 \mathrm{~nm}$. 图中 $\mathrm{TiO}_{2}$ UFP 的粒径约为 $1.5 \mathrm{~nm}$, 粒子呈现出自下往上聚集长大的趋势. 相 当多的粒子之间明显存在由毛细引力作用使其聚集排列的痕迹, 局部区域表现出六角排列结 构. 此外, 对比于图 3(a) 与图 2 可发现两图形貌上有类似之处, 但尺寸上有区别. 初步判断为 粒子聚集排列也受到基底一定诱导的作用. 由 $A$ 点转向靠近落滴处的 $B$ 点观察, 获得图象为 图 3(b) 所示, 可见到同 $A$ 类似的结构, 不同的是粒子的典型粒径为约 $3 \mathrm{~nm}$. 此外, 液面毛细 作用促使粒子团聚的迹象不明显了. 比较 $A, B$ 两点的情形可知, $B$ 点处的液膜厚度大于 $A$ 点处的, 其蒸发完所需的时间较长, 因而在蒸发期间粒子可能得以进一步聚集长大. 继续移 向更靠近落滴处的 $C$ 点观察, 扫描范围为 $68 \mathrm{~nm} \times 68 \mathrm{~nm}$, 得到图 3(c). 由图可见, 此处的粒子粒 径更大, 约 $10 \mathrm{~nm}$. 粒子排列的有序性明显不如前两处的. 由此进一步向落滴位置处靠近, 还 可见到更大粒径, 如 $50 \mathrm{~nm}$ 的大颗粒. 显然,愈靠近落滴位置, 液膜的厚度越厚, 溶剂蒸发完 毕所需时间也越长, 相应粒子就生长成更大的尺寸. 此间毛细作用退为次要, 相应于 Nagayama 等人计算的第二种情况, 即液相中的粒子聚集, 其热运动作用促使粒子相互碰撞团 聚的可能性更大. 依据以上的观察分析, 我们认为相距落滴处一定距离之外, 毛细作用下 $\mathrm{TiO}_{2}$ UFP 随溶液在 $\mathrm{HOPG}$ 上铺展的过程实际上伴随着溶剂不断蒸发, 以及 $\mathrm{TiO}_{2}$ 粒子长大的过 

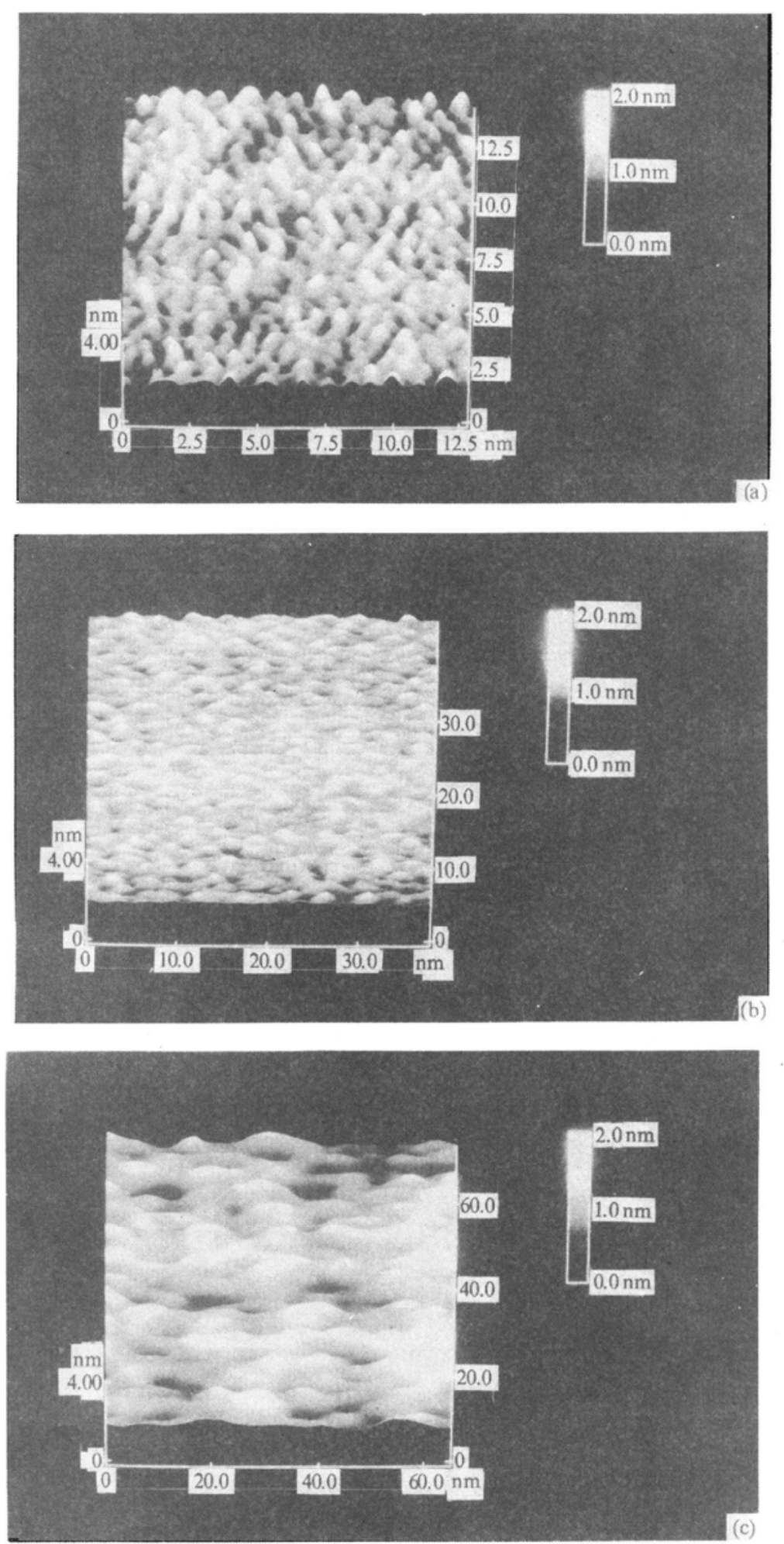

图 3 液膜铺展及毛细作用排列 $\mathrm{TiO}_{2} \mathrm{UFP}$ 膜表面结构的 $\mathrm{AFM}$ 图象 (a) (c) 分别对应于图 1(b) 中的 $A \sim C$ 点处的观察 


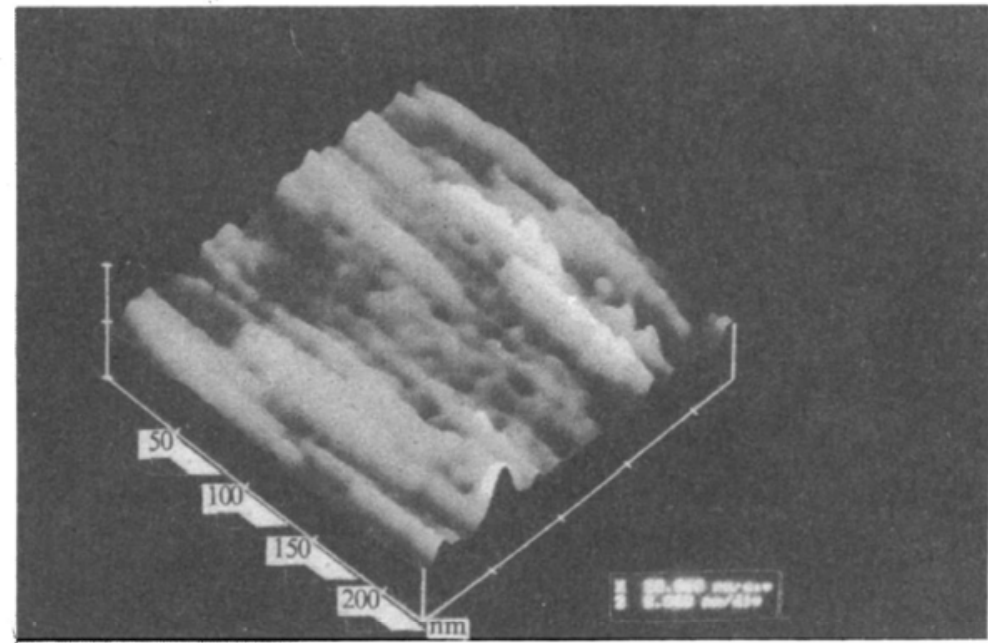

图 4 旋转涂覆 $\mathrm{TiO}_{2}$ UFP 膜表面结构的 AFM 图象

程. 最终排列 $\mathrm{TiO}_{2}$ UFP 的结构, 包括粒子的尺寸, 取决于 $\mathrm{TiO}_{2}$ 溶胶液的浓度、溶液在基底上 铺展的速度、溶剂蒸发的速率、时间以及基底条件等. 研究如何控制这些综合因素, 有可能获 得一定面积的 $\mathrm{TiO}_{2}$ 粒子有序排列的功能薄膜.

\section{3 与旋涂 $\mathrm{TiO}_{2}$ UFP 薄膜的对比}

为了进行对比, 我们采用同样浓度的 $\mathrm{TiO}_{2}$ UFP 溶胶液旋转涂覆制备得到相应的 $\mathrm{TiO}_{2}$ 薄 膜, 对其进行 AFM 观察, 如图 4 所示. 尽管采用的是同样新解理的 HOPG 表面, 但其图象展 示出明显因旋转离心造成的起伏结构, 凸起部分同其他部分一样都显现出粒子的细节. $\mathrm{TiO}_{2}$ UFP 排列的有序性明显不如依据毛细作用排列的结果. 此外, 旋涂的 $\mathrm{TiO}_{2}$ 粒子粒径为 $20 \mathrm{~nm}$ 左右, 在旋转过程中粒子也都不同程度地长大了.

\section{3 小结}

通过毛细作用使 $\mathrm{TiO}_{2}$ UFP 溶胶液在 $\mathrm{HOPG}$ 原子级的粗䊁度表面上铺展, 可获得 $\mathrm{TiO}_{2}$ 粒子很好排列的二维结构. 随着溶胶液铺展成膜, $\mathrm{TiO}_{2 . .}$ UFP 不断聚集长大, 薄膜中不同位置 处的 $\mathrm{TiO}_{2}$ 粒径与溶液浓度、该处液膜厚度以及相应溶剂蒸发时间等条件有关. 通过综合控制 这些条件,可获得较大面积窄粒径分布及有序排列的 $\mathrm{TiO}_{2} \mathrm{UFP}$ 二维超结构.

\section{参考 文 献}

1 Luscombe J H. Current issues in nanoelectronic modelling. Nanotechnology, 1993, 4(1): 1 20

2 Banyai L, Koch S W. Semiconductor Quantum Dots. Singapore: World Scientific Publishing Co Pte Ltd, 1993. $205 \sim 210$

3 Brown W D, Grannemann W W. C-V characteristics of metal-titanium dioxide-silicon capacitors. Solid State Electron, 1978, 21: $837 \sim 846$

4 Harris L A. A titanium dioxide hydrogen detecter. J Electrochem Soc, 1980, 127(12): $2657 \sim 2662$

5 Williams L M, Hess D W. Structural prorerties of titanium dioxide films deposited in an of glow discharge. J Vac Sci Technol, 1983, Al(4): $1810 \sim 1816$ 
6 深耀春, 陆祖宏, 韦 铌. 具有大比表面积的 $\mathrm{TiO}_{2}$ 超微粒半导体电极研究. 科学通报, 1994, 39(24): $2238 \sim 2240$

7 Zou B, Xiao L, Li T. Absorptien red shift in $\mathrm{TiO}_{2}$ ultrathin particles with surfacial dipole layer. Appl Phys Lett, 1991, 59 (15): $1826 \sim 1828$

8 王晓慧, 王子坑, 李 暨. 胶溶法合成 $\mathrm{TiO}_{2}$ 超微粒子. 材料科学进展, 1992, 6(6): 533 537

9 Denkov N D, Velev O D, Kralcheusky P A et al. Mechanism of formation of two-dimensional crgstals from latex particles on substrates. Langmuir, 1992, 8(12): 3183

10 Tersoff J. Anocmalous corrugations in scanning tunneling microscopy: imaging of indivduaal states. Phys Rev Lett, 1986, 57: $440 \sim 443$ 